\title{
LA QUESTION DE L'OCÉAN ARCTIQUE ENTRE TROPISME JURIDIQUE ET 'WISHFUL THINKING'
}

\author{
Claudia Cinelli* \\ Professeur \\ Université de Séville \\ claudia@us.es
}

Reçu: 1 avril 2011 / Accepté: 6 mai 2011

RESUMÉ: Cet article aborde la question de l'Océan Arctique à travers le phénomène $\mathrm{du}$ tropisme juridique. Dans une perspective historique et contemporaine, l'article analyse les différents changements d'orientation de l'ordre juridique international en réponse à l'application controversée des critères ordinaires en matière d'établissement de la souveraineté étatique à cet espace marin historiquement couvert essentiellement de glace, et aujourd'hui touché par une progressive fonte de cette dernière à cause du réchauffement terrestre. A la lumière du phénomène du dégel naturel, l'Océan Arctique a acquis une nouvelle position centrale d'importance internationale. Regardant l'avenir avec confiance et en faisant preuve d'un certain wishful thinking, cette étude se conclut dans l'espoir d'une réponse positive de la part de l'ordre international au stimulus toujours croissant de la coopération et de la solidarité nécessaires entre États arctiques et États non arctiques, afin de trouver un juste équilibre entre les intérêts particuliers des États et ceux collectifs de la Communauté internationale.

RESUM: Aquest article es refereix a la qüestió de l'Oceà Àrtic a través del fenomen del tropisme legislatiu. En aquest sentit, analitza, en perspectiva històrica i contemporània, les diferents orientacions de l'ordenament jurídic internacional en

\footnotetext{
* L'auteur souhaite remercier les Professeurs Juan Antonio Carrillo Salcedo et Joaquín Alcaide Fernández pour leur inestimable appui et leurs précieux enseignements. Des remerciements affectueux vont également à Camille Bergmann pour la révision du français.
} 
resposta a la controvertida aplicació dels criteris ordinaris dels que depèn la reivindicació de la sobirania estatal sobre aquest espai marítim que, històricament cobert principalment de gel, es veu avui, en canvi, colpejat per una retirada progressiva d'aquest a causa de l'escalfament de la terra. Des de la perspectiva que implica el fenomen emergent del desgel natural, l'Oceà Àrtic assumeix una nova posició de rellevança en el pla internacional. De cara al futur, amb una actitud basada en el wishful thinking, l'estudi es conclou amb l'esperança d'una resposta positiva per part de l'ordenament internacional enfront de l'estímul cada vegada més gran derivat d'una cooperació necessària de caràcter solidari entre els estats àrtics i no àrtics a la recerca d'un equilibri entre els interessos particulars dels estats i dels col-lectius de la comunitat internacional.

RESUMEN: Este artículo abarca la cuestión del Océano Ártico a través del fenómeno de tropismo legislativo. En este sentido, analiza, en perspectiva histórica y contemporánea, las distintas orientaciones del ordenamiento jurídico internacional en respuesta a la controvertida aplicación de los criterios ordinarios de los que depende la reivindicación de la soberanía estatal sobre este espacio marítimo que, históricamente cubierto principalmente de hielo, se ve hoy, en cambio, golpeado por una progresiva retirada del mismo a causa del calentamiento terrestre. A la luz del emergente fenómeno del deshielo natural, el Océano Ártico está asumiendo una nueva posición de relevancia en el plano internacional. De cara al futuro con una actitud basada en el wishful thinking, el estudio se concluye con la esperanza de una repuesta positiva por parte del ordenamiento internacional frente al estímulo cada vez mayor derivado de una necesaria cooperación de marcado carácter solidario entre los Estados Árticos y no Árticos en la búsqueda de un equilibrio entre los intereses particulares de los Estados y los colectivos de la Comunidad Internacional.

ABSTRACT: This article examines the question of the Arctic Ocean through the phenomenon of legislative tropism. The article specifically analyses the different manifestations, from an historical and contemporary perspective, of the international system when it has been used to challenge the controversial application of the standard criteria applied when a State makes a claim of sovereignty over formerly ice-covered 
marine territory, which is today being consumed by the gradual receding of the ice itself due to global warming. In the light of this persisting phenomenon of this natural dissipation, the Arctic Ocean is assuming a new central position of international relevance. Looking toward the future with an attitude of wishful thinking, the study ends with the hope of a positive answer which could arise from the requisite solidarity and cooperation between the Arctic State and non-Arctic States in their attempts to find equilibrium between the individual State's interests and those of the International Community.

MOTS CLÉS : Océan Arctique — Droit de la Mer — Théorie des Secteurs — Iles de Glace.

PARAULES CLAU: Oceà Àrtic — Dret del mar — Teoria dels sectors — Illes de gel.

PALABRAS CLAVES: Océano Ártico - Derecho del Mar - Teoría de los Sectores — Islas de Hielo.

KEYWORDS: Arctic Ocean — Law of the Sea — Sector Theory — Ice Islands. 
Sommaire: I. Introduction. II. La question juridique de l'Océan Arctique dans une perspective historique. 1. La question juridique de l'Océan Arctique dans la première moitié du XXème siècle. 2. La question juridique de l'Océan Arctique entre gel et dégel politique de la guerre froide. III. L'Océan Arctique à la lumière du droit international contemporain. 1. Quelques remarques sur la délimitation des plateaux continentaux dans l'Océan Arctique. 2. Quelques remarques sur la question juridique relative au Passage Nord-Est et au Passage Nord-Ouest. 3. La nécessité d'un régime juridique pour les iles de glace. IV. Conclusion. V. Bibliographia.

\section{INTRODUCTION}

L'on peut parler de tropisme ${ }^{1}$ juridique lorsque l'ordre juridique change d'orientation en réponse aux différentes nécessités de la société, par essence en constante évolution, et qu'il en résulte une modification dans l'équilibre des sources formelles et matérielles.

La question juridique de l'Océan Arctique peut être considérée comme un exemple approprié de tropisme juridique.

L'Océan Arctique — historiquement couvert essentiellement de glace, laquelle s'est toutefois retirée au fil des ans et continue aujourd'hui de se retirer à cause du changement climatique - occupe un bassin approximativement circulaire entre les terres d'Amérique du Nord, d'Asie, et d'Europe ${ }^{2}$. Bien que désigné couramment par l'expression « Océan Arctique » (le plus petit océan existant), pour ses dimensions et sa position intercontinentale stratégique, il a été dénommé par certains en plusieurs occasions Mer Méditerranée du Nord ${ }^{3}$.

Aujourd'hui comme par le passé, l'inapplication à cet espace marin, présentant des caractéristiques sui generis, des critères ordinaires en matière d'établissement de la

\footnotetext{
${ }^{1} \mathrm{Du}$ grec tropos, le terme tropisme désigne en biologie une réaction d'orientation ou de mouvement orientée, causée par des agents physiques ou chimiques. A titre d'illustration, l'on cite souvent l'exemple des plantes d'appartement qui, pour satisfaire une nécessité vitale, se courbent en s'orientant vers la lumière provenant de la fenêtre, et s'en trouvent déformées. Par extension, ce terme désigne toute réaction élémentaire à une cause extérieure.

${ }^{2}$ Il existe divers critères pour délimiter la Région Arctique, dont notamment celui de la ligne du cercle polaire arctique par $66^{\circ} 33^{\prime}$ de latitude nord et celui de la limite des arbres qui coïncide avec la ligne isotherme de $10^{\circ}$ centigrades du mois le plus chaud de l'année, à savoir juillet. Voir, DOLLOT, R., "Le droit international des espaces polaires", RCADI, Vol. 75, t. II, 1949, pp. 115-200, p. 124; MOUTON, M.W., "The international regime of the polar regions", RCADI., Vol. 107, t. III, 1962, pp. 169-286, p. 176; PHARAND, D., "The legal status of the Arctic regions", RCADI, Vol. 163, t. II, 1979, pp. 49-116, p. 59. Pour d'autres discussions voir, HAYTON, R.D, "The polars problems and international law", in AJIL., Vol. 52, No. 4, 1958, pp. 746 -765, p. 747; KEKITALO, E.C.H., Negotiating the Arctic. The construction of an international region, Routledge, New York/Londres, 2004, p. x et pp. 30-35.

${ }^{3}$ Hayton, "The polars...", op. cit., p. 747 et p. 749; MOUTON, "The international...", op. cit., p. 230; HOLMES, J.W., Foreword, in MacDONALD, R. St.J., , The Arctic Frontier, University of Toronto Press, Toronto, 1966.
} 
souveraineté étatique engendre une certaine insécurité juridique et donne lieu à des revendications diverses de la part de plusieurs Etats.

Ainsi, le début du XXème siècle déjà connut un phénomène de tropisme juridique. Le régime traditionnel de liberté de la haute mer se modifia en effet en faveur de la souveraineté de l'État côtier, fondée, d'une part, sur l'application de l'analogie glacies firma - terra firma, et, d'autre part, sur une certaine interprétation de la soi-disant théorie des secteurs.

Dans les années suivant immédiatement la Seconde Guerre Mondiale, le phénomène du tropisme juridique, notamment à l'égard de l'Océan Arctique, acquit une connotation négative. Pendant la période de la guerre froide, on ne trouva pas de réponse normative positive aux exigences de maintien de la paix et de sécurité collective dans cet océan stratégique qui sépare les côtes des États-Unis de celles de l'actuelle Fédération de Russie.

Pendant ces difficiles années de gel tant politique que naturel, l'Océan Arctique sortit discrètement de la scène internationale pour y revenir seulement au début du XXIème siècle, lorsque, les dégels politique et naturel, joints à un progrès technologique vertigineux, ravivèrent la séculaire obsession du territoire ${ }^{4}$ envers cette région.

Aujourd'hui, l'on assiste ainsi à un nouveau phénomène de tropisme juridique. Les cinq États côtiers arctiques - Canada, Danemark, États-Unis, Fédération de Russie et Norvège - tentent en effet de modifier à leur profit les institutions juridiques relatives à la délimitation des espaces marins, et cela tout particulièrement en ce qui concerne la délimitation des plateaux continentaux, le statut juridique des Passages du Nord-Est et du Nord-Ouest qui relient l'Océan Atlantique à l'Océan Pacifique, ainsi que la question de l'absence de régime juridique applicable aux îles de glace flottant dans l'Océan Arctique.

Dans le monde contemporain, caractérisé par une forte interdépendance, l'Océan Arctique constitue un espace d'importance internationale en raison de sa position géopolitique stratégique et de son fort potentiel économique en termes d'exploitation des ressources naturelles et de contrôle des routes commerciales, sans parler de son rôle en matière environnementale.

\footnotetext{
${ }^{4}$ SCELLE, G., Obsession du territoire. Essai d'étude réaliste de droit international, cité par P.M. Dupuy, Droit international public, Dalloz, Paris, 8ème éd., 2006, p. 747.
} 
Aussi peut-on espérer, en faisant preuve d'un certain wishful thinking, que l'ordre juridique international réponde de manière positive au stimulus toujours croissant de la coopération et de la solidarité nécessaires entre États arctiques et États non-arctiques qu'il s'agisse de grandes, moyennes ou petites puissances -, afin de trouver un juste équilibre entre les intérêts particuliers des États et ceux collectifs de la Communauté internationale $e^{5}$.

\section{LA QUESTION JURIDIQUE DE L'OCÉAN ARCTIQUE DANS UNE PERSPECTIVE HISTORIQUE}

En avril 1909, l'Océan Arctique se présenta aux yeux de Robert Peary - qui commandait l'une des deux premières expéditions américaines à parvenir jusqu'à l'extrême $\operatorname{Nord}^{6}$ - comme presque entièrement formé de "glace dure, à peine couverte de neige et que marquait de taches d'un bleu de saphir la glace d'eau douce des lacs de l'été précédent"7.

$\mathrm{Au}$ vu des variations constantes de surface et d'épaisseur de la glace dans l'Océan Arctique au fil des saisons, est surgie la question juridique suivante: "Y aura-t-il pour la glace un régime différent de celui de la terre et de celui de l'eau ?"8.

1. La question juridique de l'Océan Arctique dans la première moitié du XXème siècle

Il est notoire qu'au début du XXème siècle primait une vision en surface de la mer et que le régime de la haute mer se réduisait principalement à la liberté de navigation ${ }^{9}$.

\footnotetext{
5 "C'est en définitive d'une sorte de condominium plural qu'elles [les régions polaires] doivent être l'objet: elles doivent devenir une possession commune de tous les membres de la famille des nations. [...] En raison de l'interdépendance et de la solidarité économiques des Etats, l'un des fondements du droit des gens moderne est la coopération, sur la base de l'égalité juridique, de tous les pays du monde: chaque fois que dans le droit international une situation nouvelle apparaît, le règlement doit en être cherché dans l'intérêt général et non pas dans l'intérêt égoïste de telle ou telle nation déterminée. La communauté internationale ne peut pas être un mot vide de sens." [FAUCHILLE, P., Traité de droit international public, 2ème Partie, Livre Premier, Rosseau \& C ${ }^{\circ}$, Paris, 1925, p. 658-659 (italiques ajoutés)].

${ }^{6}$ BROWN SCOTT, J., "Arctic exploration and international law", AJIL,Vol. 3, 1909, pp. 928-941.

${ }^{7}$ Cité par WAULTRIN, R., (DOLLOT, R.), "Le problème de la souveraineté des Pôles", RGIDP, Tome XVI, 1909, pp. 649-660, pp. 654-55.

${ }^{8}$ Ibid., p. 655.

${ }^{9}$ GIDEL, G., Le droit international public de la mer, Tome I, Mellottée, Paris, 1932, p.125 ss.
} 
Cette liberté était toutefois réduite dans l'Océan Arctique, en particulier durant l'hiver, à cause de la présence de la glace. Tandis que la formation de la celle-ci empêchait de facto la libre navigation dans l'Océan Arctique, elle rendait en revanche possible l'occupation de ce territoire, soulevant dès lors la question de la souveraineté étatique sur ce qui paraissait être glacies firma ${ }^{10}$.

En conséquence, le problème se posait de savoir si l'on devait considérer la glacies firma dans la haute mer comme terra firma - res nullius, susceptible d'appropriation de la part de n'importe quel État, ou comme mare libre - res communis, soustraite à tout forme d'appropriation étatique.

Pour une partie de la doctrine, la solution à ce problème résidait dans l'extension ipso facto de la limite de la mer territoriale -à l'époque généralement estimée de trois milles marines - à l'espace de mer glacée adjacente à la côte, en tant qu'accession naturelle de la terra firma. Selon cette position doctrinale, et conformément à la logique juridique de l'accessorium sequitur principale, la formation de la glace au-delà des trois milles de la côte relevait de la souveraineté de l'Etat côtier et dérogeait donc au régime traditionnel de liberté de la haute mer ${ }^{11}$.

Toutefois, la qualification de glacies firma ne paraissait pas tout à fait appropriée du moment que la glace en question formait, selon les conditions climatiques et les courants marins, des espaces "à géométrie variable"12.

Aussi, face aux difficultés soulevées par l'analogie terra firma - glacies firma, une autre partie de la doctrine s'en tenait-elle à la thèse selon laquelle l'Océan Arctique, en tant que haute mer, constituait une res communis conformément à la Law of the

\footnotetext{
${ }^{10}$ BOGGS, S.W., The polar regions: geographical and historical data for consideration in a study of claims to sovereignity in the Arctic and Antartic regions, 1933, reédite par William S. Hein \& Co., Inc., New York, 1990, p. 30 ss.

${ }^{11}$ En 1904, considérant les questions juridiques soulevées par la construction d'un Casino bâti sur la glace au-delà de la limite de la mer territoriale de l'Alaska (États-Unis d'Amérique), ROLLAND, L., affirmait que "quand il y a congélation de la limite considérée comme normale, la mer territoriale s'étend, ipso facto, pour autant, tant que les glaces ne sont point fondues." ["Etats -Unis d'Amérique-Alaska. Maison de jeu établie sur les glaces au-delà de la limite des eaux territoriales", RGDIP, Tome XI, 1904, pp. 340345, p. 344]. Voir aussi WAULTRIN: "terre ferme ou glace immobile, déclare-t-il, les pôles sont susceptibles d'appropriation, par suite permettent l'exercice d'une souveraineté; mer libre ou glace flottante soumise aux perpétuelles dérives des courants polaires, les pôles ne sont pas susceptibles d'appropriation et constituent un domaine maritime, une res communis" ["Le problème... "op. cit., pp. 655-656].

${ }^{12}$ BEDJAOUI, M., "Le Statut de la glace en droit international", in M. RAMS MONTALDO (dir.), Le droit international dans un monde en mutation. En hommage au professeur Eduardo Jiménez Aréchaga, Fundación de Cultura Universitaria, Montevideo, 1994 pp. 713-729, p. 721.
} 
Nations $^{13}$ et ne dérogeait donc pas, en tant que tel, au régime traditionnel de la haute mer.

A ces deux premières théories relatives au régime applicable à l'Océan Arctique s'ajoute encore celle découlant de l'élaboration de la soi-disant théorie des secteurs.

Considérant l'inapplicabilité à la Région Arctique des critères ordinaires en matière d'établissement de la souveraineté territoriale (terra nullius - animus occupandi), le sénateur canadien Poirier proposa, lors de la session du Parlement d'Ottawa du 19 février 1907 , de diviser cette région en secteurs ${ }^{14}$.

L'idée était de faire correspondre chaque secteur à un espace de forme triangulaire ayant pour sommet le Pôle Nord et pour base une ligne reliant les points situés à l'extrême Est et à l'extrême Ouest des côtes de chaque État arctique.

Selon la théorie de Poirier, la souveraineté de chaque Etat arctique devait alors s'étendre sur toutes les terres et les îles (et non sur la mer) situées géographiquement à l'intérieur du secteur correspondant ${ }^{15}$.

Par la suite, une partie de la doctrine se fonda sur une interprétation extensive de la théorie originaire de Poirier pour établir une théorie dérivée, selon laquelle le secteur était considéré comme un prolongement (artificiel) contigu des terres arctiques ayant pour point de convergence le Pôle Nord, de sorte que la souveraineté étatique s'étendait non seulement aux terres et îles, mais également à la haute mer arctique située à l'intérieur dudit secteur, à laquelle s'appliquait ainsi le régime de la mer territoriale ${ }^{16}$.

\footnotetext{
13 "And so the rules of the Law of Nations that recognize the freedom of the high seas, would seem to apply naturally to a moving and shifting substance like the North Polar Sea ice at all points beyond the customary three-mile limit from the shore" [BALCH, T.W., "The Arctic and Antarctic regions and the Law of Nations", AJIL., Vol.2, No.2, 1910, pp. 265-275, p. 266]. Voir aussi par le même auteur, "Les Régions Arctiques et Antarctiques et le droit international", RDI., 2ème série, Vol. XII, 1910, pp. 434442, p. 434.

${ }^{14}$ Les passages les plus significatifs de cette discussion dans le compte rendu du Sénat sont cités par R.WAULTRIN (R. DOLLOT), "La question de la souveraineté des terres Arctiques", RGIDP, Tome XV, 1908, 3ème partie, pp. 401-423, p. 413.

${ }^{15}$ A la question: "Qu'entend-on effectivement par secteur? ", Dollont répondait qu'il s'agissait d'un "procédé de répartition des terres polaires entre les Etats qui se trouvent placés au voisinage de ces terres, le secteur constituant un triangle sphérique dont le sommet est au pôle, dont les côtés sont les méridiens et la base une côte ou des parallèles" [Dollont, "Le droit...", op. cit., p. 127 (italiques ajoutés)]. En ce sens voir, DIEZ DE VELASCO, M., Instituciones de derecho internacional público, Tecnos, Madrid, 10ème éd., 2007, p. 558.

${ }^{16}$ LAKHTINE, W., affirmait que "sovereignity should attach to the Polar States over the Arctic Ocean within their sectors of attraction" ["Rights over the Arctic", AJIL., Vol. 24, No. 4, 1930, pp. 703-717, p. 713]. En ce sens voir aussi, CONFORTI, B., Diritto internazionale, Editoriale Scientifica, Naples, 7ème éd., 2006, p. 287.
} 
Fondée sur le principe de contiguïté/adjacence et dérogeant au critère de l'animus occupandi, la théorie des secteurs consacrait ainsi une nouvelle forme pacifique d'établissement de la souveraineté sur des espaces territoriaux (et maritimes) présentant des caractéristiques géophysiques sui generis.

L'examen de la pratique des premières années révèle cependant que la théorie (originaire et dérivée) des secteurs n'a pas été élevée au rang de règle générale. A cet égard, il suffira de rappeler deux exemples significatifs de certaines déclarations des gouvernements russe et norvégien, ainsi que la jurisprudence internationale.

Ainsi, s'agissant des déclarations gouvernementales, l'on peut relever notamment que la Norvège reconnut en 1930 la souveraineté canadienne sur les îles Sverdrup, tout en précisant que cette reconnaissance était "in no way based on any sanction whatever of what is named sector principle" ${ }^{\text {}}$. De même, en 1926, le Gouvernement soviétique revendiqua sa souveraineté uniquement sur les terres et sur les îles (mais non sur la haute mer) situées dans le secteur qui lui correspondait ${ }^{18}$.

Quant à la jurisprudence internationale, il convient de citer la célèbre affaire de l'Île de Palmas, où l'arbitre Max Huber affirma, en 1928, que le principe de contigüité entraînait une certaine insécurité quant à la délimitation des espaces et ne constituait dès lors pas un critère approprié pour trancher les différends en matière de souveraineté territoriale $^{19}$. Par ailleurs, dans l'affaire du Statut juridique du territoire du Groenland Oriental, la Cour Permanente de Justice Internationale jugea qu'il n'y avait pas lieu de déroger aux conditions consacrées par le droit international classique en matière d'établissement de la souveraineté. Afin de tenir compte des conditions naturelles particulières du Groenland, elle tempéra toutefois le principe de l'animus occupandi par celui de l'animus possessionis et considéra qu'un exercice sporadique dans le temps et

\footnotetext{
${ }^{17}$ Cf. Treaty Series No. 25, H.M.S.O., Londres, 1931, p. 3875.

18 "Decree of the Presidium of the Central Executive Committee of the USSR", 15 avril 1926, citè par Mounton, "The international...", op.cit., p. 197.

${ }^{19}$ Ille de Palmas (Pays-Bas/Etats-Unis), 4 avril 1928, R.I.A.A. /R.S.A., Vol. II, pp. 829-871, p. 855. Il faut remarquer que pareille position a été maintenue tout récemment par la Cour Internationale de Justice (CIJ), laquelle "ne fonde pas ses conclusions sur l'adjacence" dans l'affaire Différend territorial et maritime entre le Nicaragua et le Honduras dans la mer des Caraïbes (Nicaragua/Honduras), arrêt du 8 octobre 2007, Rôle General No. 120, \$164.
} 
isolé dans l'espace de l'autorité étatique avait été suffisant, en l'espèce, pour établir la souveraineté du Danemark sur le Groenland ${ }^{20}$.

En conclusion, il est clair que la théorie (originaire et dérivée) des secteurs ne donna pas naissance à une règle généralement reconnue comme étant le droit.

D'autre part, la classification traditionnelle mer territoriale - haute mer fut l'objet d'une profonde transformation qui, à partir de 1945, mena à un processus articulé de codification du droit international maritime.

La première conférence de codification en la matière conduisit à l'adoption des quatre Conventions de Genève de $1958^{21}$. Parmi celles-ci, la Convention sur la haute mer mit un frein aux revendications sui generis de souveraineté des Etats arctiques sur les espaces marins, en reconnaissant le régime de liberté de la haute $\operatorname{mer}^{22}$, lequel descend, tout en lui apportant certaines limites, du principe traditionnel de liberté appliqué par la doctrine classique à la res communis omnium.

\section{La question juridique de l'Océan Arctique entre gel et dégel politique de la guerre froide}

Après la Seconde Guerre Mondiale, les obstacles de facto à la liberté de navigation du début du XXème siècle furent franchis grâce aux développements technologiques qui rendirent possible la navigation aérienne, en surface et sous-marine de l'Océan Arctique.

Pendant la période de la guerre froide, les dangereuses activités of defense ${ }^{23}$ menées tant par les États-Unis que par l'URSS rendaient toutefois le régime de libre navigation dans la haute mer arctique fort risqué. Aussi la question juridique de l'Océan Arctique se déplaça-t-elle, à la fin des années cinquante, sur le plan du maintien de la paix et de la

\footnotetext{
${ }^{20}$ CPIJ, Statut juridique du territoire du Groenland Oriental (Danemark/Norvège), arrêt du 5 avril 1933, série $A / B$ No. 53, pp. 45-46.

${ }^{21}$ Convention sur la mer territoriale et la zone contiguë; faite à Genève le 29 avril 1958, entrée en vigueur le 10 septembre 1964 - Nations Unies, Recueil des Traités, Vol. 516; Convention sur la haute mer; faite à Genève le 29 avril 1958, entrée en vigueur le 30 septembre 1962 - Nations Unies, Recueil des Traités, Vol. 450; Convention sur la pêche et la conservation des ressources biologiques de la haute mer, faite à Genève le 29 avril 1958, entrée en vigueur le 20 mars 1966 - Nations Unies, Recueil des Traités, Vol. 559; Convention sur le plateau continental, faite à Genève le 29 avril 1958, entrée en vigueur le 10 juin 1964 - Nations Unies, Recueil des Traités, Vol. 499.

${ }^{22}$ En vertu de l'Article 2

${ }^{23}$ HAYTON, "Polar...", op. cit., p. 746.
} 
sécurité collective. La question était de savoir si la haute mer devait être soumise à un régime juridique de neutralisation et de désarmement en raison de sa position géographique stratégique entre les deux superpuissances.

Le 21 avril 1958, l’URSS demanda au Conseil de Sécurité de prendre des mesures pour mettre fin au passage sur la région arctique d'avions américains armés de bombes atomiques et à hydrogène ${ }^{24}$. À leur tour, le 28 avril de la même année, les États-Unis demandèrent au Conseil de Sécurité d'instituer dans la région arctique un système international d'inspection ${ }^{25}$. Comme on pouvait l'imaginer, le Conseil de Sécurité demeura bloqué par les vetos croisés.

La situation était si délicate que l'on évitait toute occasion d'attirer l'attention internationale sur la région arctique. Fait significatif, la Troisième Année Polaire Internationale $(1957-58)^{26}$ fut exceptionnellement appelée Année Géophysique Internationale, en référence aux activités de coopération internationale scientifique visant spécifiquement le continent antarctique. $\mathrm{Au} \mathrm{vu}$ des différents intérêts internationaux sur ce continent, celui-ci fut doté, l'année suivante, d'un régime international sui generis, sanctionné par le Traité sur l'Antarctique de $1959^{27}$.

Ainsi, tandis que l'attention portée par la Communauté internationale à l'Antarctique jetait les bases du concept juridique de patrimoine commun de l'humanité ${ }^{28}$, l'Océan Arctique sortait, pour sa part, discrètement de la scène internationale.

\footnotetext{
${ }^{24}$ Cf. Doc. S/3993, 21 avril 1958

26 - Cf. Doc. S/3995, 28 avril 1958.

${ }^{26}$ La tradition des années polaires remonte à il y a 125 ans. Lancée initialement par l'Organisation Internationale Météorologique d'alors, la première année polaire internationale fut inaugurée en 1882-83, et fut suivie, cinquante ans après, par la deuxième en 1932-33. À vingt cinq années de distance, le Conseil International des Sciences (CIS) et l'Organisation Météorologique Mondiale (OMM) proclamèrent la troisième année polaire internationale, dénommée officiellement Année Géophysique Internationale, 1957-58. Cinquante ans après, l'OMM et le CIS inaugurèrent l'actuelle quatrième Année Polaire Internationale (2007-2008). Cette nouvelle Année polaire est placée sous les auspices du réchauffement climatique, qui marque de son empreinte les zones arctiques. Voir le site web officiel: htpp://www.ipy.org.

${ }^{27}$ Voir, inter alia, GUYER, R., "The Antartic system", RCADI., Vol. 139, t. II, 1973, pp. 149-226; FRANCIONI, F., SCOVAZZI, T., International law for Antartica, Kluwer Law International, La Haye, 1992; FRANCIONI, F., "Le conservation et la gestion des ressources de l'Antarctique", RCADI., Vol. 260, 1996, pp. 239-404.

${ }^{28}$ Cf. par exemple, Res. AG 40/156, 21 janvier 1986. Pour des critiques et commentaires voir, inter alia, PUREZA, J.M.,, El patrimonio común de la humanidad. ¿Hacía un derecho internacional de la solidaridad?, Trotta, Madrid, 2002.
} 
Au fil des ans, le gel provoqué par ces fortes tensions bipolaires céda peu à peu le pas au phénomène opposé de dégel politique, caractérisé par une coopération interétatique croissante.

De nombreuses négociations multilatérales furent alors consacrées, dans le cadre d'un lent mais constant développement du droit international, à la réalisation des intérêts collectifs de la Communauté internationale, notamment en matière de désarmement ${ }^{29}$ et de gestion des ressources naturelles du sol et du sous-sol marin ${ }^{30}$.

De profonds changements virent ainsi le jour et donnèrent lieu, inter alia, à une révision substantielle du droit international maritime avec, pour point d'orgue, l'adoption en 1982 de la Convention de Montego Bay (ci-après la Convention de 1982) ${ }^{31}$, suivant le régime juridique solidariste du patrimoine commun de l'humanité, entrée en vigueur en 1994 avec l'Accord relatif à l'application de sa partie XIème portant sur la gestion des ressources sous-marines au-delà des limites de la juridiction nationale.

La Convention de 1982, à participation presque-universelle ${ }^{32}$ (parmi les cinq États côtiers arctiques - Canada, Danemark, Norvège, Fédération de Russie et États-Unis seulement ce dernier ${ }^{33}$ ne l'a pas encore ratifiée ${ }^{34}$ ) et codifiant en grande partie le droit coutumier, consacre un seul article aux zones recouvertes par la glace dans le cadre de la protection et de la préservation du milieu marin ${ }^{35}$.

Malgré le dégel politique de la guerre froide et l'impulsion donnée par le leader soviétique Gorbatchev en faveur de l'exercice d'activités of cooperation dans la région

\footnotetext{
${ }^{29}$ Il suffit de rappeler l'institution en 1968 du Comité sur les utilisations pacifiques des fonds des mers et des océans au-delà des limites de la juridiction nationale [Res. AG 2467 (XXIII), 21 décembre 1968].

${ }^{30}$ Il suffit de rappeler l'affirmation du concept de patrimoine commun de l'humanité dans la Déclaration des principes régissant le fond des mers et des océans, ainsi que leur sous-sol, au-delà des limites de la juridiction nationale [Res. AG 2749 (XXV), 17 décembre 1970].

${ }^{31}$ Les quatre Conventions de Genève de 1958 se révélèrent inadéquates face aux nouvelles exigences économiques et aux nouvelles capacités d'exploitation des ressources marines. Aussi firent-elles l'objet d'une révision substantielle lors de la Troisième Conférence, qui eut lieu à Montego Bay du 1974 au 1982 et qui mena à l'adoption d'une Convention unique signée à Montego Bay le 10 décembre 1982.

${ }^{32}$ À la date du 2011 la Convention compte 161 ratifications, y compris celle de la Union européenne du 1 avril 1998

${ }^{33}$ Pour certaines discussions sur la position americaine, voir BANDOW, D., Don't resurrect the Law of the Sea Treaty, Cato Institute, Washington D.C., 2005; "Intelligence brief: Arctic scramble leads Washington to reconsider law of the sea" in Power and interest news reports, 26 octobre 2007, consultable à la page: http://www.pinr.com/.

${ }^{34}$ Dates de ratification respectivement et en ordre chronologique: Norvège, 24 juin 1996; Fédération de Russie, 12 mars 1997; Canada, 7 novembre 2003; Danemark, 16 novembre 2004.

${ }^{35} \mathrm{Cf}$. l'article 234
} 
$\operatorname{arctique}^{36}$, la question juridique de l'Océan Arctique demeurait en marge de la scène internationale.

Ce n'est qu'au début du XXIème siècle et en raison des phénomènes du réchauffement de la Terre et du dégel naturel que l'Océan Arctique acquit une nouvelle position centrale d'importance internationale.

\section{L'OCÉAN ARCTIQUE À LA LUMIÈRE DU DROIT INTERNATIONAL CONTEMPORAIN}

Si au début du siècle dernier la question de l'Océan Arctique se rapportait à la présence de la glace, aujourd'hui, au contraire, elle est centrée autour des conséquences de la diminution progressive de la glace causée par le changement climatique. L'augmentation de la température est un phénomène particulièrement pertinent dans la Région Arctique, car il en améliore l'accessibilité, jusqu'alors très réduite ${ }^{37}$.

Aussi l'Océan Arctique se trouve-t-il actuellement au carrefour de revendications ${ }^{38}$ fondées sur la Convention de 1982 de la part des États arctiques, visant à étendre leurs droits d'exploration et d'exploitation des ressources naturelles sur les plateaux continentaux aussi bien que leur souveraineté sur les Passages Nord-Est et Nord-Ouest dont on estime qu'ils seront facilement navigables dans un futur proche.

Il convient en outre de remarquer que le phénomène du réchauffement de la planète contribue à une augmentation de la separatio de la glace de la barrière de glace, conduisant à la formation d'un nombre toujours plus grand de soi-disant îles de glace flottant librement dans l'Océan Arctique. Comme l'augmentation de la température

\footnotetext{
${ }^{36}$ Le discours prononcé par le leader Gorbatchev à Mourmansk en 1987, dans le cadre général de la politique de glasnost, est généralement considéré comme le moment (Mourmansk Momentum) qui signa le début du processus plus ample, aujourd'hui encore en cours, de construction d'une région internationale arctique. Voir, OSHERENKO, G., et YOUNG, O.R., The age of the Arctic. Hot conflits and cold realities, Cambridge University Press, Cambridge, 1989, p. 240 ss; ISSRAELIAN, E., "L'initiative de Gorbatchev à Mourmansk et les mesures de restauration de la confiance dans l'Arctique", Etudes Internationales, Vol. XX, No.1, 1989, pp. 61-70; YOUNG, O.R., Creating regimes. Arctic accords and international governance, Cornell University Press, Ithaca/Londres, 1998, pp. 172173; KEKITALO, Negotiating..., op. cit., p. 42 ss; HØNNELAND, G., et STOKKE,O.L., International cooperation and Arctic governance. Regime effectiveness and Northern region building, Routledge, Londres, 2007, p. 164 ss.

${ }^{37}$ Voir, "Nasa's jet propulsion laboratory, California Institute of Technology - Nasa examines Arctic sea ice changes leading to record law in 2007", consultable à la page http://www.nasa.gov/vision/earth.

${ }^{38}$ L.B., "Carrefour des revendications", RGDIP, Vol. CXI, 2007, pp. 900-901
} 
accroît la vulnérabilité de la glace, ces îles ont toutefois une durée d'existence plus courte que par le passé. Les îles de glace n'ont jamais été prises en considération du point de vue du droit international, bien qu'elles paraissent soulever des questions juridiques très importantes.

\section{Quelques remarques sur la délimitation des plateaux continentaux dans l'Océan}

\section{Arctique $^{39}$}

Le 20 décembre 2001 la Fédération de Russie informa la Commission des limites du plateau continental ${ }^{40}$ (ci-après la Commission des limites) de la délimitation du rebord externe de sa marge continentale dans, inter alia, l'Océan Arctique au-delà de 200 milles marins des lignes de base à partir desquelles est mesurée la largeur de la mer territoriale ${ }^{41}$.

Conformément à l'article 50 du Règlement intérieur de la Commission des limites (ciaprès Règlement intérieur) ${ }^{42}$, la demande de la Fédération de Russie fut notifiée aux États membres des Nations Unies, et en particulier aux États parties à la Convention.

Bien qu'ils ne fussent alors pas Parties à la Convention, le Canada et le Danemark réagirent immédiatement. Ils déclarèrent ainsi qu'ils ne disposaient pas des informations nécessaires pour évaluer la demande de la Fédération de Russie, mais précisèrent

\footnotetext{
${ }^{39}$ Pour une discussion initiale, voir, inter alia, OUDE ELFERINK, A.G., "The Outer continental shelf in the Arctic: the application of article 76 of the LOS Convention in a regional context" in OUDE ELFERINK, A.G., et ROTHWELL, D.R., (dir.), The law of the sea and polar maritime delimitation and jurisdiction, Martinus Nijhoff Publishers, La Haye/New York/Londres, 2001, pp. 139-156; SKARIDOV, A.S., et SKARIDOVA, M.A., "Legal aspects of Russian perspectives on continental shelf in the Arctic"; MAYER, L., JAKOBSSON, M., HALL, J., "Challenges of collecting Law sea of the sea data in the Arctic", in NORDQUIST, M.H., MOORE, J.N., SKARIDOV, A.S., (dir.), International energy policy, the Arctic and the law of the sea, Center for Oceans Law and Policy, Martinus Nijhoff Publishers, Leiden/Boston, 2005, pp. 79-98 et 125-140, respectivement; KUNOY, B., "A new Arctic conquest: The Arctic outer continental margin", Nordic J.I.L., Vol. 76, 2007, pp. 465-480; JARASHOW, M., RUNNELS, M.B., SEVENSON, T., "UNCLOS and the Arctic: The path of least resistance", in Fordham I.L.J., Vol. 30, 2007, pp. 1387-1652; BENITAH, M., "Russia's claim in the Arctic and the vexing issue of ridges in UNCLOS", ASIL Insight, Vol. 11, No. 27, 2007.

${ }^{40}$ Voir article 76.8 et Annexe II de la Convention de 1982.

${ }^{41}$ Le résumé de la communication russe ne pas disponible, voir le site web de la Commission des limites: http://www.un.org/Depts/los/clcs.

${ }^{42}$ La dernière version du Règlement intérieur de la Commission des limites (CLCS/40) fut approuvée lors de la treizième session New York, 26-30 avril 2004. Plus d'informations sur le site web de la Commission de limites, cit.
} 
néanmoins que l'impossibilité de formuler à ce stade des observations n'impliquait pas de leur part un consentement exprès ou tacite à la position russe ${ }^{43}$.

La Norvège, pour sa part, informa la Commission des limites d'un "différend maritime" avec la Fédération de Russie relatif à la Mer de Barents ${ }^{44}$ et, le 27 novembre 2006, présenta une communication concernant la délimitation du rebord externe de sa marge continentale au-delà des 200 milles marins, notamment dans l'Océan Arctique ${ }^{45}$.

Si le Danemark, l'Islande et la Fédération de Russie ne soulevèrent aucune objection particulière à cette communication norvégienne, ils se réservèrent toutefois la possibilité de saisir la Commission des limites dans le futur et/ou de conclure avec la Norvège des accords de délimitation ${ }^{46}$.

Mais le débat sur la délimitation du plateau continental dans l'Océan Arctique ne touche pas seulement les Etats arctiques. En effet, la mission permanente de l'Espagne auprès des Nations Unies indiqua au Secrétariat Général qu'elle avait envoyé, le 2 mars 2007, une note à la Norvège, dans laquelle elle exprimait sa position concernant les espaces marins relatifs à l'archipel du Spitzberg (Svalbard). En tant qu'État partie au Traité de Paris relatif à l'Archipel du Spitzberg de 1920 (ci-après Traité de Paris de 1920) ${ }^{47}$, l'Espagne revendiquait ainsi les droits d'exploitation des ressources du plateau continental y relatif, tant à l'intérieur qu'à l'extérieur des 200 milles $^{48}$.

\footnotetext{
${ }^{43}$ Voir respectivement pour le Canada et le Danemark, Doc. CLCS.01.2001.LOS/CAN, 26 février 2002; Doc. CLCS.01.2001.LOS/DNK, 26 février 2002. A noter également que les États-Unis mirent en doute la crédibilité de la Commission de limites et de la Convention de 1982, Doc. CLCS.01.2001.LOS/USA. Consultable sur le site web de la Commission des limites, cit.

${ }^{44}$ Cf. Doc. CLCS.01.2001.LOS/NOR, 2 avril 2002. Consultable sur le site web de la Commission des limites, cit.

${ }^{45}$ Plateau continental Communication de la Norvège relative à certains secteurs de l'océan Arctique, de la mer de Barents et de la mer de Norvège, R és u m é. Consultable sur le site web de la Commission des limites, cit.

${ }^{46}$ Voir respectivement pour le Danemark, l'Islande et la Fédération de Russie, Doc. Ref. No. 119.N.8, 27 janvier 2007; Partial Submission of the Government of the Kingdom of Denmark together with the Government of the Faroes in the area north of the Faroe Islands, 29 avril 2009 (uniquement en anglais); Doc. Ref: FNY07010008/97.B.512., 27 janvier 2007; The Icelandic Continental Shelf. Partial Submission to the Commission on the Limits of the Continental Shelf pursuant to article 76, paragraph 8 of the United Nations Convention on the Law of the Sea in respect of the Agir Basin area and Reykjanes Ridge, 29 avril 2009 (uniquement en anglais); Doc. Ref. $\mathrm{N}^{\circ}$ 82/n (traduction en anglais du russe non officiel), 21 février 2007. Enfin, voir, Recommandations de la Commission des limites du plateau continental concernant la demande faite par la Norvège au sujet de zones de l'océan Arctique, de la mer de Barents et de la mer de Norvège le 27 novembre 2006. Consultable sur le site web de la Commission des limites, cit.

${ }^{47}$ Société de Nations - Recueil des Traités, Vol. 2 No. 41.

${ }^{48}$ Doc. Ref : 184JR/ot, 3 mars 2007. Consultable sur le site web de la Commission des limites, cit
} 
En réponse, le 28 mars 2007 la Mission permanente de la Norvège auprès des Nations Unies réaffirma sa souveraineté sur l'archipel aux termes de l'article $1 \mathrm{du}$ Traité de Paris de 1920. En outre, elle rappela que l'interprétation de ce dernier ne saurait influencer d'aucune manière l'interprétation et l'application des règles consacrées par la Convention de 1982 et les compétences attribuées à la Commission des limites ${ }^{49}$.

La Commission des limites, aux sens de l'article 76.8 de la Convention de 1982, est compétente pour adresser des recommandations sur les questions concernant la fixation des limites extérieures du plateau continental. Ces limites, une fois fixées par l'État côtier sur la base de ces recommandations, sont considérées comme définitives et obligatoires. En cas de différends maritimes, conformément à l'article 5 b) du Règlement intérieur, les demandes présentées à la Commission des limites et les recommandations que celle-ci approuve doivent être prises en considération sans préjudice de la position des États parties au différend.

S'agissant ainsi des discussions actuelles autour de la délimitation des plateaux continentaux de l'Océan Arctique, la Commission des limites - conformément à son modus operandi - institua une sous-commission ayant la tâche d'examiner la demande russe $^{50}$, demanda l'envoi de documentation supplémentaire ${ }^{51}$, adopta certaines recommandations ${ }^{52}$ et invita la Fédération de Russie à reformuler sa propre demande.

Etant donné le grand nombre de différends internationaux portant sur la délimitation des plateaux entre États adjacents ou entre États qui se sont face, les organes juridictionnels ou arbitraux internationaux accumulèrent progressivement un certain bagage jurisprudentiel qui pourrait contribuer à rationaliser les négociations futures entre les États arctiques en vue de l'élaboration d'accords de délimitation équitables.

A cet égard, l'arrêt de principe rendu en 1969 par la CIJ sur la délimitation du plateau continental de la mer du $\operatorname{Nord}^{53}$ - dont la ratio fut reprise à l'article 83 de la Convention et développée par la jurisprudence internationale postérieure - paraît suggérer l'idée suivante. Une fois fixés les points pour établir la ligne de base de la mer

\footnotetext{
${ }^{49}$ Mission permanente de la Norvège auprès des Nations Unies, 28 mars 2007. Consultable sur le site web de la Commission des limites, cit

${ }^{50}$ Cf. Doc. CLCS/29, para. 9.

${ }^{51}$ Cf. Doc. CLCS/32, para. 16; Un Doc. CLCS/34 para 5.

${ }^{52}$ Voir le Rapport du Secrétaire général, à la Cinquante-septième session de l'Assemblé Générale, point 25 a) de l'ordre du jour Les océans et le droit de la mer, UN Doc. A/57/57/Add.1, § 38-41.

${ }^{53}$ Plateau continental de la mer du Nord, arrêt, C.I.J. Recueil 1968, p.3.
} 
territoriale, il conviendra d'appliquer des critères correctifs à la règle générale de l'équidistance afin d'aboutir à un résultat équitable ${ }^{54}$.

Dans l'attente d'une délimitation définitive, les États concernés devront, suivant l'article 83.3 de la Convention, conclure des arrangements provisoires.

Mais qu'entend-on par "arrangements provisoires"? En attendant un accord définitif, un Etat peut-il prétendre à l'utilisation exclusive des zones de plateaux controversées?

Concernant les événements les plus récents, il faut rappeler qu'en juillet-août 2007 la Fédération de Russie, avant même d'avoir reformulé sa demande auprès de la Commission, organisa une expédition au Pôle Nord, au cours de laquelle pour la première fois dans l'Histoire deux bathyscaphes Mir en immersion profonde se posèrent sur le fond de l'Océan Arctique. Ils y plantèrent un drapeau russe en titane et y laissèrent également une capsule contenant un message à l'attention des générations futures, lequel rappelle les valeurs de solidarité et d'étroite collaboration entre tous les chercheurs de différentes générations œuvrant pour la réalisation du bien commun de l'humanité ${ }^{55}$.

Toutefois et pour conclure, force est de constater que les discussions actuelles sur la délimitation des plateaux de l'Océan Arctique ne prennent pas suffisamment en compte les espaces restants pour la Zone et la gestion pour l'humanité des ressources minières in situ ${ }^{56}$ où, selon les termes de M. Lachs, "the role of equity [which] becomes more important within and without the law" $" 57$.

\footnotetext{
54 "En ce qui concerne le tracé d'une frontière maritime unique, la Cour a clairement indiqué à diverses reprises que, lorsqu'il s'agit d'établir une ligne couvrant plusieurs zones de juridiction qui coïncident, la méthode dite des principes équitables et des circonstances pertinentes peut utilement être appliquée, cette méthode permettant également d'aboutir dans ces zones maritimes à un résultat équitable", C.I.J., Différend territorial et maritime entre le Nicaragua et le Honduras dans la mer des Caraïbes (Nicaragua/Honduras), cit., § 271.

${ }^{55}$ Le message aux générations futures laissé par l'expédition russe au Pôle Nord (Message de Ĉilingarov) est cité en langue italienne par M. De Bonis, "Le mani sul polo", in Il clima dell'energia, Limes Rivista Italiana di Geopolitica, No. 6, 2007, pp. 185-192, p. 192.

${ }^{56}$ PHARAND, D., "L'Arctique et l'Antarctique: Patrimoine commun de l'humanité ?", Annales de droit aérien et spatial, Vol. VII, 1982, 415- 430.

${ }^{57}$ LACHS, M., "Ouverture du colloque" in R.J. DUPUY, La Gestion des Ressources pour l'Humanité: le droit de la mer, Académie de droit international de la Haye, Colloque 29-31 octobre 1981; Martinus Nijhoff Publishers, La Haye, 1982, pp. 3-5, p. 5.
} 


\section{Quelques remarques sur la question juridique relative au Passage Nord-Est et au Passage Nord-Ouest ${ }^{58}$}

L'Océan Arctique et l'Océan Pacifique sont reliés par le Passage Nord-Est et par le Passage Nord-Ouest, longeant respectivement les eaux de la Fédération de Russie et du Canada. Historiquement accessibles seulement pendant quelques mois par an, on estime que ces Passages seront pleinement navigables dans un avenir proche ${ }^{59}$.

D'où la nécessité de conférer à ces Passages un statut juridique définitif, en tenant compte des particularités propres à chacun.

Il se peut tout d'abord que, soit la Fédération de Russie, soit le Canada revendiquent les eaux correspondant respectivement au Passage Nord-Est et Nord-Ouest, en tant qu' eaux intérieures sur la base d'un titre historique. Une telle solution serait tout à l'avantage des deux Etats susmentionnés, mais porterait atteinte à tous les autres, car elle permettrait à la Fédération de Russie et au Canada d'éviter de voir leur souveraineté sur ces Passages restreinte par le droit de passage inoffensif reconnu dans les mers territoriales ${ }^{60}$.

$\mathrm{Si}$, au contraire, lesdits Passages étaient considérés comme faisant partie respectivement de la mer territoriale de la Fédération de Russie et du Canada, ces Etats seraient alors tenus d'y respecter le droit de passage (inoffensif) des navires étrangers, pourvu qu'il soit continu et rapide ${ }^{61}$.

\footnotetext{
58 Voir http://maps.grida.no/go/graphic/northern-sea-route-and-the-northwest-passage-compared-withcurrently-used-shipping-routes; Pour une discussion initiale sur la question, voir MCKINNON, B., Arctic sovereignty and the Northwest Passage, The Vancouver Bar Association, Vancouver, 1986; STEWART, W.P., The impact of international law on Canada's claim to sovereignty in the Arctic archipelago with particular reference to "historical waters" and ice, Scott Polar Research Institute, University of Cambridge, Thèse de doctorat, 1991; ELLIOT-MEISEL, E.B., Arctic diplomacy: Canada and the United States in the Northwest Passage, Lang, New York, 1998; TIMCHENKO,L., "The Northen Sea Route: Russian management and jurisdiction over navigation in Arctic seas", in A.G. OUDE ELFERINK ET D.R. ROTHWELL (dir.), The law of the sea..., op. cit., pp. 269 -293; R.D. BRUBAKER, "The Russian Arctic Straits", in G.J. MANGONE, International straits of the world, Martinus Nijhoff Publishers, Leiden/Boston, 2005, pp. 25 ss; D. PHARAND, "The Arctic waters and the Northwest Passage: A final revisit", ODIL, Vol. 38, N. 1, 2007, pp 3-69; D. McRae, "Arctic sovereignty? What is at stake?", BTH., Vol. 64, No.1, 2007, pp. 1-23; M. JARASHOW, M.B. RUNNELS ET T. SEVENSON, "UNCLOS and the Arctic: The path of least resistance", op. cit.; KRASKA, J., "The law of the sea and the Northwest Passage", IJMCL, Vo. 2, No. 22, 2007, pp. 257-282.

${ }^{59}$ Le Passage Nord-Ouest s'est entièrement ouvert pour la première fois pendant l'été 2007. Voir, inter alia, "Taking the Northwest Passage", BBC News, 15 octobre 2007

${ }^{60}$ Il faut avoir bien à l'esprit que l'article 8.2 de la Convention de 1982 prévoit la situation suivante : "Lorsque le tracé d'une ligne de base droite établie conformément à la méthode décrite à l'article 7 inclut dans les eaux intérieures des eaux qui n'étaient pas précédemment considérées comme telles, le droit de passage inoffensif prévu dans la Convention s'étend à ces eaux".
}

${ }^{61}$ Articles 17 et suivants de la Convention de 1982. 
Etant donné, cependant, la composante géographique (une zone de mer qui relie deux espaces marins dans lesquels est en vigueur la liberté de navigation) et la éventuelle composante fonctionnelle (l'utilisation pour la navigation internationale) ${ }^{62}$, rien n'empêche que les deux Passages soient qualifiés de détroits servant à la navigation internationale.

Dans ce cas, le droit de passage inoffensif des navires étrangers, qui ne peut être gêné ou suspendu, se transforme en véritable droit de transit. En effet, les détroits peuvent, à la différence de la mer territoriale, être librement survolés et traversés par des sousmarins sans que ceux-ci aient l'obligation de naviguer en surface ${ }^{63}$.

Cela dit, la pratique relative aux Passages Nord-Est et Nord-Ouest ne s'est pas encore clairement dessinée et la discussion relative aux intérêts politiques et économiques est encore trop incertaine pour permettre de se prononcer définitivement sur les solutions juridiques aux sens de la Convention.

De toute façon, il est toujours possible de déroger aux dispositions de la Convention de 1982 - régime d'eaux intérieures, régime de passage inoffensif, régime des détroits - en faveur d'une internationalisation de ces deux Passages. Cette dernière solution, négociée de manière à assurer une juste balance des intérêts, nécessiterait une étroite coopération entre États Arctiques et non Arctiques, au vu des modifications substantielles du trafic international maritime que l'ouverture des deux Passage suppose dans un avenir proche.

\section{La nécessité d'un régime juridique pour les iles de glace}

L'île de glace est un terme arctique et évoque l'idée d'un territoire sujet à occupation de la part de l'homme. Provenant généralement de la rupture d'un plateau de glace arctique, les îles de glace sont couramment définies comme de larges surfaces flottantes de glace d'eau douce, et correspondent à ce que, en Antarctique, on appelle iceberg à forme tabulaire. Le droit international présente un vacuum normatif par rapport aux îles de glace.

En pratique, les îles de glace furent arbitrairement occupées par la Fédération de Russie et les Etats-Unis et utilisées comme stations de dérive à des fins scientifiques maritimes,

\footnotetext{
${ }^{62}$ Affaire du Détroit de Corfou, arrêt du 9 avril 1949, C.I.J. Recueil 1949, p. 4.

${ }^{63}$ Articles 37 et suivants de la Convention de 1982.
} 
ou plus rarement (étant donné précisément leur lente mais constante dérive) comme station de forage.

Au vu de cette pratique, le Professeur canadien Pharand, lors de son cours à l'Académie de droit international de La Haye en $1973^{64}$, eut recours à l'analogia legis et posa la question suivante : "Could an ice island be considered as a natural island, an artificial island or a ship?"65. Il opta pour l'analogie avec les navires "to the extent it is applicable",66.

Cette solution reflète une approche territoriale, d'après laquelle l'île de glace relève de la souveraineté territoriale de l'État côtier tant qu'elle se trouve à l'intérieur de ses frontières maritimes, devient une res nullius quand elle est laissée inoccupée à la dérive en haute mer, et acquiert le statut juridictionnel d'un navire du moment qu'elle est occupée par un Etat en vertu de la règle générale prior in tempore potior in iure.

Il en va différemment si, au lieu de retenir une approche territoriale, l'on considère les îles de glace comme une forme d'utilisation de la mer ${ }^{67}$. Alors en effet, la manière dont les îles de glace sont utilisées est déterminante pour définir leur statut juridique. A cet égard, deux possibilités différentes se dégagent.

En premier lieu et conformément à la pratique, l'île de glace peut être utilisée comme un lieu où exercer des activités de recherche scientifique maritime à des fins licites et pacifiques, ou, lorsque l'île se trouve en face d'un plateau continental, elle peut être temporairement utilisée come station de forage. En tant que station de recherche ou station de forage, l'île de glace peut se voir appliquer, mutatis mutandis, respectivement les dispositions sur la recherche maritime prévues par la partie XIIIème de la Convention de 1982, ou celles relatives aux îles artificielles et installations consacrées à l'article 60 de cette dernière.

En deuxième lieu et même s'il n'existe encore aucune pratique dans ce sens, l'île de glace pourrait être considérée comme une forme d'exploitation de la mer, en tant que

\footnotetext{
${ }^{64}$ PHANRAD, "The legal status...", op. cit.

${ }^{65}$ Ibid., p. 93.

66 " $[\ldots]$ it would seem that the most practical and realistic way to deal with the problem is to consider them as ships and apply their legal regime to the extent that it is applicable", Ibid., p. 100.

67 A propos des îles de glace, MOUTON s'exprima ainsi: "Now what is the status of these drifting camps? They are not to be assimilated with ships, because they are not registered as such. It is a form of use of the high sea" ["The international...", op. cit., p. 209].
} 
ressource épuisable d'eau douce flottant sur sa surface ${ }^{68}$. Cette solution présente un grand intérêt théorique, surtout maintenant que le réchauffement climatique tend à accélérer la dislocation des plateaux de glace et, partant, la séparation des îles de glace, tout en réduisant la durée d'existence de ces dernières ${ }^{69}$.

La vulnérabilité de la glace rend toujours plus précaire l'utilisation des îles de glace comme station de recherche ou station de forage. Regardant vers l'avenir, l'on peut imaginer que, grâce à l'irrépressible progrès technologique, il sera possible d'en éviter la perte en faveur de leur exploitation comme ressource d'eau douce ${ }^{70}$, pour autant toutefois que l'on évite de tomber dans une commercialisation immodérée de ce bien commun $^{71}$.

Etant donné l'affirmation, au sein de la communauté internationale, d'une certaine tendance solidariste ${ }^{72}$ et considérant les défis soulevés en droit international par la pénurie croissante d'eau douce sur la Terre ${ }^{73}$, l'on peut, à notre sens, envisager de proclamer les îles de glace comme îles de toute l'humanité.

L'internationalisation des îles de glace en tant que ressources épuisables d'eau douce pourrait être réalisée par le biais d'une forme de coopération étroite entre États, en vue de la protection des intérêts collectifs de la Communauté internationale.

En définitive, la reconnaissance d'un intérêt collectif supérieur pourrait fonder l'établissement d'un régime international destiné à assurer un certain équilibre entre,

\footnotetext{
${ }^{68}$ JOYNER, C., "The status of ice in international law", op.cit., pp. $29-49$.

${ }^{69}$ Ainsi par exemple, en août 2005, le Plateau de glace d'Ayles s'est rompu brusquement, engendrant la création d'une nouvelle île de glace (Gouvernement du Canada, Environnemental: http://iceglaces.ec.gc.ca). Celle-ci s'est à son tour fracturée en deux parties en octobre 2007. Voir, "Arctic ice island breaks in half", $B B C$ News, 1 octobre 2007.

${ }^{70}$ Voir C. JOYNER, "The status of Ice in International law", op.cit. En transposant cette logique sur les icebergs dans l'Antarctique, F. TROMBETTA-PANIGADI écrit que "icerberg must be think and large enough to be exploited usefully and economically as a resource of fresh water." ["The explotation of Antartic Icerbarg in international law" in F. FRANCIONI et T. SCOVAZZi, International law..., op. cit., pp. 225-257, p. 231].

${ }^{71}$ À titre d'exemple voir le lien japonais au internet shop de glace de l'Alaska: http://item.rakuten.co.jp. En général, sur les problèmes causés par une commercialisation de l'eau, même dans son état solide, voir S. MC CAFFREY, "Water Scarcity: Institutional and Legal responses" in E. BRANS, The Scarcity of Water: Emerging legal and Policy Responses, Kluwer Law International, Londres, 1997, pp. 43-58, p. 57.

${ }^{72}$ Voir P.M. DUPUY, "L'unité de l'ordre juridique international: Cours général de droit international public", RCADI., Vol. 297, 2002, pp. 9-489, p. 245-268. Voir aussi J.A. CARRILLO SALCEDO, Algunas reflexiones sobre la noción de Comunidad Internacional, Anales de la Real Academia de Ciencias Morales y Políticas, Vol. LIX, No. 84, Madrid, 2007.

${ }^{73}$ Voir BOISSON DE CHAZOURNES, L., (dir.), Les ressources en eau et le droit international, L'Académie de droit International de La Haye, Martinus Nijhoff Publishers, La Haye, 2005.
} 
d'une part, la conservation (dans la mesure où celle-ci est possible) et, d'autre part, la gestion des ressources épuisables d'eau douce flottant dans l'Océan Arctique.

La question reste ouverte.

\section{CONCLUSION}

L'analyse de la question juridique de l'Océan Arctique confirme que les normes juridiques ne peuvent pas être considérées comme un phénomène autonome et isolé par rapport aux intérêts politico-économiques et aux valeurs éthico-sociales, qui, à leur tour, en conditionnent l'application et l'effectivité ${ }^{74}$.

En effet, la protection des intérêts particuliers des cinq États côtiers s'oppose à celle des intérêts collectifs de la Communauté internationale, laquelle implique une certaine convergence des normes positives et naturelles vers une prise de conscience de l'interdépendance globale de valeurs, telles que la dignité humaine, la paix, ainsi que la sécurité environnementale, économique et militaire ${ }^{75}$.

À ce stade, il existe ainsi deux stimuli induisant opposés - intérêts particuliers versus intérêts collectifs ${ }^{76}$-, en réponse auxquels l'ordre juridique est appelé à se modifier, en s'orientant dans l'une ou l'autre direction, afin de résoudre la question de l'Océan Arctique.

Dans l'attente que ce phénomène de tropisme juridique se réalise, cette étude se conclut par une prospective future fondée sur le wishful thinking.

Depuis le début des années quatre-vingt-dix, l'on assiste à un processus politique en faveur de la construction de la région arctique, promu par les Grandes Puissances Arctiques $^{77}$ animées par un esprit d'internationalité de caractère fonctionnel ${ }^{78}$.

\footnotetext{
${ }^{74}$ Voir, DE VISSCHER, C., Théories et réalités en droit international public, Pedone, Paris, 4ème éd., 1970.

${ }^{75}$ TANZI, A., Introduzione al diritto internazionale contemporaneo, Cedam, Padue, 2010, p.17.

${ }^{76}$ Sur la base de cette logique d'opposition entre intérêts particuliers des États et intérêts collectifs de la Communauté internationale, voir ALCAIDE FERNANDEZ, J., "The contemporary high seas fisheries regime: Not a free-for-all, but... How free?, in CASADO RAIGÓN (dir.), L'Europe et la mer (pêche, navigation et marine environnement), Bruylant, Bruxelles, pp. 315-331.

${ }^{77}$ L'expression est utilisée par G. HØNNELAND et O.L. STOKKE pour décrire les acteurs politiques principaux (à leur avis, Etats-Unis, Fédération de Russie et Union européenne) dans le cadre du processus de coopération internationale actuellement en cours dans la Région Arctique. [International cooperation..., op. cit., p. 6 ss].
} 
Sous le signe de la quatrième Année Polaire Internationale 2007-2008 en cours, l'on peut espérer que l'ère de gloire des grands empires fasse désormais partie du passé et que l'esprit d'internationalité de caractère fonctionnel se joigne à celui solidariste de la Communauté internationale, afin de trouver une éventuelle solution évolutive de caractère normatif à la question Arctique, dans la synthèse dialectique entre sources juridiques formelles et matérielles qui gouverne le processus d'évolution normative permanente, conformément à l'adage: hominum causa omne jus constitum est.

\section{BIBLIOGRAPHIE}

ALCAIDE FERNÁNDEZ, J., "The contemporary high seas fisheries regime: Not a free-for-all, but... How free?, in CASADO RAIGÓN (dir.), L'Europe et la mer (pêche, navigation et marine environnement), Bruylant, Bruxelles, pp. 315-331.

BALCH, T.W., "The Arctic and Antarctic regions and the Law of Nations", AJIL., Vol.2, No.2, 1910, pp. 265-275.

-"Les Régions Arctiques et Antarctiques et le droit international”, RDI., 2ème série, Vol. XII, 1910, pp. 434-442..

BANDOW, D., Don't resurrect the Law of the Sea Treaty, Cato Institute, Washington D.C., 2005; "Intelligence brief: Arctic scramble leads Washington to reconsider law of the sea" in Power and interest news reports, 26 octobre 2007, consultable à la page: http://www.pinr.com/.

BEDJAOUI, M., "Le Statut de la glace en droit international", in M. RAMS MONTALDO (dir.), Le droit international dans un monde en mutation. En hommage au professeur Eduardo Jiménez Aréchaga, Fundación de Cultura Universitaria, Montevideo, 1994 pp. 713-729, p. 721.

BENITAH, M., "Russia's claim in the Arctic and the vexing issue of ridges in UNCLOS”, ASIL Insight, Vol. 11, No. 27, 2007.

\footnotetext{
${ }^{78}$ Durant ces vingt dernières années, trois importants organes intergouvernementaux furent institués: l'Arctic Council (http://www.arctic-council.org/), le Barents Euro Arctic Region (http://www.beac.st/) et le Council of the Baltic Sea State (http://www.cbss.st/). En outre, de nombreuses organisations nongouvernementales furent fondées. Enfin, au sein du Conseil Arctique, l'Université de l'Arctique (http://www.uarctic.org/) fut dotée d'importants projets de recherche en matière de protection de l'environnement, commerce, industrie, santé, éducation et culture.
} 
BOGGS, S.W., The polar regions: geographical and historical data for consideration in a study of claims to sovereignity in the Arctic and Antartic regions, 1933, réédité par William S. Hein \& Co., Inc., New York, 1990.

BOISSON DE CHAZOURNES, L., (dir.), Les ressources en eau et le droit international, L'Académie de droit International de La Haye, Martinus Nijhoff Publishers, La Haye, 2005.

BROWN SCOTT, J., "Arctic exploration and international law", AJIL., Vol. 3, 1909, pp. 928-941.

BRUBAKER, R.D., "The Russian Arctic Straits", in G.J. MANGONE, International straits of the world, Martinus Nijhoff Publishers, Leiden/Boston, 2005, pp. 25 ss;

CAFFREY, M.C., "Water Scarcity: Institutional and Legal responses" in E. BRANS, The Scarcity of Water: Emerging legal and Policy Responses, Kluwer Law International, Londres, 1997, pp. 43-58.

CARRILLO SALCEDO, J.A., "Algunas reflexiones sobre la noción de Comunidad Internacional", Anales de la Real Academia de Ciencias Morales y Políticas, Vol. LIX, No. 84, Madrid, 2007.

CONFORTI, B., Diritto internazionale, Editoriale Scientifica, Naples, 7ème éd., 2006, p. 287.

DIEZ DE VELASCO, M., Instituciones de derecho internacional público, Tecnos, Madrid, 10ème éd., 2007

DOLLOT, R., "Le droit international des espaces polaires", RCADI, Vol. 75, t. II, 1949, pp. 115-200;

DUPUY, P.M., “L'unité de l'ordre juridique international: Cours général de droit international public", RCADI., Vol. 297, 2002, pp. 9-489, p. 245-268.

ELliOT-MEISEL, E.B., Arctic diplomacy: Canada and the United States in the Northwest Passage, Lang, New York, 1998.

FAUCHILlE, P., Traité de droit international public, 2ème Partie, Livre Premier, Rosseau \& $C^{\mathrm{o}}$, Paris, 1925.

FRANCIONI, F., SCOVAZZI, T.,, International law for Antartica, Kluwer Law International, La Haye, 1992. 
FRANCIONI, F., "Le conservation et la gestion des ressources de l'Antarctique", RCADI., Vol. 260, 1996, pp. 239-404.

GIDEL, G., Le droit international public de la mer, Tome I, Mellottée, Paris, 1932.

GUYER, R., “The Antartic system”, RCADI., Vol. 139, t. II, 1973, pp. 149-226.

HAYTON, R.D, “The polars problems and international law”, in AJIL., Vol. 52, No. 4, 1958, pp. 746-765.

HØNNELAND, G., et STOKKE, O.L., International cooperation and Arctic governance. Regime effectiveness and Northern region building, Routledge, Londres, 2007.

ISSRAELIAN, E.,"L'initiative de Gorbatchev à Mourmansk et les mesures de restauration de la confiance dans l'Arctique", Etudes Internationales, Vol. XX, No.1, 1989.

JARASHOW, M., RUNNELS, M.B., SEVENSON, T., "UNCLOS and the Arctic: The path of least resistance", in Fordham I.L.J., Vol. 30, 2007, pp. 1387-1652.

KEKITALO, E.C.H., Negotiating the Arctic. The construction of an international region, Routledge, New York/Londres, 2004.

KRASKA, J., "The law of the sea and the Northwest Passage", IJMCL, Vo. 2, No. 22, 2007, pp. 257-282.

KUNOY, B., "A new Arctic conquest: The Arctic outer continental margin", Nordic $J I L$, Vol. 76, 2007, pp. 465-480.

L.B., “Carrefour des revendications”, R.G.D.I.P., Vol. CXI, 2007, pp. 900-901

LACHS, M., "Ouverture du colloque" in R.J. DUPUY, La Gestion des Ressources pour l'Humanité: le droit de la mer, Académie de droit international de la Haye, Colloque 29-31 octobre 1981; Martinus Nijhoff Publishers, La Haye, 1982, pp. 3-5, p. 5.

LAKHTINE, W., "Rights over the Arctic”, AJIL., Vol. 24, No. 4, 1930, pp. 703-717.

M.H. NORDQUIST, J.N. MOORE ET A.S. SKARIDOV (dir.), International energy policy, the Arctic and the law of the sea, Center for Oceans Law and Policy, Martinus Nijhoff Publishers, Leiden/Boston, 2005.

McDONALD, R. St.J., , The Arctic Frontier, University of Toronto Press, Toronto, 1966. 
MCKINNON, B., Arctic sovereignty and the Northwest Passage, The Vancouver Bar Association, Vancouver, 1986.

McRAE, D., “Arctic sovereignty? What is at stake?”, B.T.H., Vol. 64, No.1, 2007, pp. $1-23$;

MOUTON, M.W., "The international regime of the polar regions", RCADI., Vol. 107, t. III, 1962, pp. 169-286.

OSHERENKO, G., et YOUNG, O.R., The age of the Arctic. Hot conflits and cold realities, Cambridge University Press, Cambridge, 1989.

OUDE ELFERINK, A.G., et ROTHWELL, D.R., (dir.), The law of the sea and polar maritime delimitation and jurisdiction, Martinus Nijhoff Publishers, La Haye/New York/Londres, 2001.

PHARAND, D., “L'Arctique et l'Antarctique: Patrimoine commun de l'humanité ?”, Annales de droit aérien et spatial, Vol. VII, 1982, 415- 430.

- "The legal status of the Arctic regions", RCADI, Vol. 163, t. II, 1979, pp. 49-116.

-"The Arctic waters and the Northwest Passage: A final revisit”, ODIL, Vol. 38, N. 1, 2007, pp 3-69;

PUREZA, J.M.,, El patrimonio común de la humanidad. ¿Hacía un derecho internacional de la solidaridad?, Trotta, Madrid, 2002.

ROLLAND, L., "Etats -Unis d'Amérique-Alaska. Maison de jeu établie sur les glaces au-delà de la limite des eaux territoriales”, RGDIP, Tome XI, 1904, pp. 340-345

STEWART, W.P., The impact of international law on Canada's claim to sovereignty in the Arctic archipelago with particular reference to "historical waters" and ice, Scott Polar Research Institute, University of Cambridge, Thèse de doctorat, 1991

TIMCHENKO,L., "The Northen Sea Route: Russian management and jurisdiction over navigation in Arctic seas", in A.G. OUDE ELFERINK ET D.R. ROTHWELL (dir.), The law of the sea..., op. cit., pp. 269 -293.

WAULTRIN, R., (DOLLOT, R.), "Le problème de la souveraineté des Pôles", RGIDP, Tome XVI, 1909, pp. 649-660, pp. 654-55.

-"La question de la souveraineté des terres Arctiques", RGIDP, Tome XV, 1908, 3ème partie, pp. 401-423, p. 413. 
YOUNG, O.R., Creating regimes. Arctic accords and international governance, Cornell University Press, Ithaca/Londres, 1998. 